BULLETIN Bulletin hispanique

HISPANIQUE Université Michel de Montaigne Bordeaux

110-1 | 2008

Varia

\title{
Michel Boeglin, Inquisición y Contrarreforma. El tribunal del Santo Oficio de Sevilla (1560-1700)
}

Jean-Pierre Dedieu

\section{OpenEdition}

1 Journals

Édition électronique

URL : https://journals.openedition.org/bulletinhispanique/687

DOI : 10.4000/bulletinhispanique.687

ISSN : 1775-3821

Éditeur

Presses universitaires de Bordeaux

Édition imprimée

Date de publication : 1 juin 2008

Pagination : 385-388

ISBN : 978-2-86781-511-9

ISSN : 0007-4640

\section{Référence électronique}

Jean-Pierre Dedieu, « Michel Boeglin, Inquisición y Contrarreforma. El tribunal del Santo Oficio de Sevilla (1560-1700) », Bulletin hispanique [En ligne], 110-1 | 2008, document 15, mis en ligne le 08 juin 2012, consulté le 18 septembre 2021. URL : http://journals.openedition.org/bulletinhispanique/687 ; DOI : https://doi.org/10.4000/bulletinhispanique.687 
qui sont métaphoriquement du côté de l'animalité ", en même temps qu'il évoque une postérité d'Alice dont il aurait été intéressant de rechercher la trace possible dans l'univers hispanique. L'inspiration psychanalytique et les concepts utilisés ne font pas partie de l'arsenal des études antérieures ce qui pique la curiosité de savoir si une telle problématique y trouverait sa place.

Au total ce livre, malgré certaines rigidités de son cadre, n’est pas la simple juxtaposition d'études séparées que sont parfois certains ouvrages collectifs. Ses fils conducteurs, adéquats aux grands traits constitutifs de la culture hispanique, se donnent à lire au fil des analyses et cela fait regretter l'absence d'une sorte de réflexion synthétique qui viendrait relayer et enrichir les efforts de l'insuffisant lecteur. A son intention également pourrait apparaître au bilan, au vu des pratiques étudiées, un état des définitions du terme " modèle ", tant sont variés les degrés de proximité avec le modèle catholique si prégnant, au-delà peut-être de la séparation des genres, choisie ici, et avec une forte pertinence descriptive, comme fil conducteur. La qualité des travaux du groupe Infantina laisse apercevoir que ce regroupement des faisceaux de réflexion arrivera à son heure et donnera de la littérature pour enfants dans les textes hispaniques une vision qui ne sera pas simplement chronologique ou thématique mais, comme on le voit dans cet ouvrage et le précédent, originalement attachée aux pratiques et aux propositions de lecture. L'Espagne n'a certes pas fourni à nos imaginaires une Alice, un Peter Pan ou un Tom Sawyer. Mais l'univers hispanique montre bien à l'œuvre la continuité d'une riche littérature d'enfance et de jeunesse dont l'oralité est une composante fondamentale et dont les déchirements d'une histoire culturelle complexe fondent souvent le parcours. Il est utile qu'un tel regroupement des travaux en lien avec les pédagogues et les créateurs vienne enrichir la connaissance et la compréhension d'un domaine sur lequel le regard de l'hispanisme peut se révéler fécond. Ce livre en témoigne. Le suivant est attendu.

Jean ALSINA

Michel Boeglin, Inquisición y Contrarreforma. El tribunal del Santo Oficio de Sevilla (1560-1700), Sevilla, Ayuntamiento de Sevilla - Ediciones Espuela de Plata, 2006, 278 + XXIV p. d'illustrations. - ISBN 84-96098-75-3.

Ce travail est une version remaniée, abrégée et recentrée de la thèse de l'auteur, publiée à Montpellier en $2003^{1}$. Il gagne beaucoup à cette refonte :

1. Michel Boeglin, L'Inquisition au lendemain du Concile de Trente: le tribunal du Saint 
la qualité éditoriale est remarquable (25 illustrations pleine page, maquette élégante et réussie); le texte, débarrassé de ses excroissances universitaires et centré sur l'essentiel, gagne en agilité et en force de conviction. Du bel ouvrage. Et de fait, un nouveau livre.

Il s'agit presque exclusivement d'une étude et d'un commentaire de l'activité de l'inquisition de Séville en matière de foi, reconstruite à partir des relations de cause et de la correspondance, puisque les procès sont perdus. Le travail conserve une facture classique, reprenant des approches et retrouvant des conclusions que l'historiographie antérieure avait déjà formulées pour l'inquisition en général et María Victoria González de Caldas retrouvées pour l'inquisition de Séville 2 . Avouerais-je préférer la version de M. Boeglin, plus ferme, plus dense et qui situe mieux les choses dans leur contexte ${ }^{3}$ ?

Nous n'aurons donc pas de révélations fondamentales. Les grands rythmes de l'activité sont confirmés: brutalité de la répression d'un noyau limité de spirituels autochtones, qui tiraient fortement vers la Réforme à la fin de années 1550 et au début des années 1560; montée en puissance par la suite des délits mineurs de vieux-chrétiens, blasphèmes, propositions diverses, et surtout campagne systématique contre la croyance que la simple fornication n'est pas un péché; répression circonstancielle de l'islamisme des morisques, des esclaves africains et des tentatives de passages en Afrique du Nord, qui acquiert une certaine importance au début du XVII ${ }^{e}$ siècle; vagues ponctuelles de répression de ce que les inquisiteurs rangent sous la dénomination d'«alumbrados» (vers 1575; vers 1625); remontée en puissance du vieux fond de commerce inquisitorial des poursuites antijudaïques à la fin du XVI et tout le XVII ${ }^{e}$ siècle. Les tournants principaux de l'histoire du tribunal sont bien marqués: la refondation de la fin des années 1550; la réorganisation du premier tiers du XVII ${ }^{e}$ siècle avec l'atténuation des poursuites contre les vieux-chrétiens et la remise au premier plan de l'activité antijudaïque.

Pour M. Boeglin, l'inquisition est avant tout une agence religieuse, chargée d'implanter les nouvelles tendances formulées par le Concile de Trente. Une désexualisation du monde sacré, en premier lieu, qui explique

Office de Sévile (1560-1700), Montpellier, Presse de l'Université de Montpellier, 2003, $678 \mathrm{p}$.

2. Victoria González de Caldas, ¿judíos o cristianos? El proceso de fe. Sancta Inquisitio, Sevilla, Universidad de Sevilla, 2000, 660 p.; González de Caldas (Victoria), El poder y su imágen. La inquisición real, Sevilla, Universidad de Sevilla, 2001, 263 p.

3. En dépit de quelques formulations un peu rapides: je ne vois pas en quoi le fait que le curé doive être ordonné pour dire la messe garantit l'orthodoxie des prédicateurs (p. 144) et je crains fort que l'auteur ait confondu inquisiteur général et doyen de l'inquisition de Séville (p. 126). 
les poursuites à propos de la simple fornication, contre les alumbrados - nous y reviendrons -, contre les confesseurs sollicitants. Une cléricalisation de l'Eglise, ensuite, qui réduit les laïcs au rang de mineurs, irresponsables de leur conduite, mais où les prêtres, perçus comme leurs leaders naturels, font l'objet d'une surveillance étroite; cléricalisation qui explique la forte concentration de la répression inquisitoriale sur les clercs: plus de $40 \%$ des protestants autochtones poursuivis entre 1560-1565 avaient reçu les ordres sacrés; plus de $80 \%$ des alumbrados, alors que la masse des dénoncés étaient des laïcs. Une division claire du monde sacré, en deuxième lieu, entre le Bien (Dieu, ses saints et ses prêtres) et le Mal (le Démon, ses suppôts et la masse des petits magiciens, sorciers de village, rebouteux et leur clientèle), qui explique la répression de la hechicería et de l'astrologie. Enfin et surtout, en troisième lieu, l'affirmation de frontières au sein de la chrétienté, indispensables à une véritable prise en main des fidèles. La frontière extérieure, l'islam, ne suffit ni à créer un sentiment d'urgence, ni a justifier une inquisition des consciences: l'altérité du non-chrétien est trop marquée pour qu'il y ait risque de confusion. Il en va autrement du nouveau-chrétien de juif ou du protestant, péril sournois, qui appelle un appareil de contrôle capable de pénétrer l'intimité des êtres.

Un point a particulièrement retenu mon attention: la répression de l'alumbradismo. M. Boeglin voit dans les pratiques que l'inquisition range sous ce nom une protestation populaire contre une religion pilotée d'en haut, une aspiration à la proximité personnelle avec la divinité, une divinité proche des soucis et des aspiration des hommes - des femmes aussi, peutêtre même surtout. Ceci permet à l'auteur d'établir une continuité entre les protestants autochtones, les alumbrados, le quiétisme, la croyance en l'inocuité de la simple fornication et de quelques autres pratiques sexuelles consenties et la sollicitation in actu confessionis. Il suggère derrière cela, bien sûr, une spécificité régionale. L'inquisition n'aime pas. Elle aime même beaucoup moins que la hiérarchie épiscopale locale, qui canalise, tolère et parfois bénit. Alors de temps en temps l'inquisition frappe un grand coup, contre l'alumbradismo ou la simple fornication, pour rappeler les limites à ne pas dépasser. La brièveté de ses accès en la matière indique bien qu'il ne s'agit pour elle que de rappeler les principes.

Les poursuites contre les alumbrados de 1623/1625 sont assez particulières pour que l'auteur s'étonne. Nous avons d'une part la proclamation d'un édit de grâce (1623) que amène près de 2000 témoignages, au point que l'inquisition, débordée, doit habiliter les dominicains de Séville à recevoir les confessions des personnes qui se présentent spontanément. Les rapports de synthèse parlent d'un monde grouillant et nombreux de frères et de dévotes 
de tous milieux sociaux, qui mènent des aventures à la fois spirituelles et affectives à fortes connotations sexuelles, milieux à l'évidence étrangers aux orientations officielles de l'Eglise du temps. Bilan : quelques condamnations à des peines qui blessent essentiellement l'honneur et la réputation de leaders choisis, et dont la notoriété ne dépasse pas, dans la plupart des cas, le cercle restreint d'un milieu clérical étroit. Cette discordance eût peut-être moins intrigué l'auteur s'il avait mieux pris en compte les observations des spécialistes italiens de l'inquisition sur les modes d'action du tribunal à partir de la seconde moitié du XVI siècle. La priorité n'est plus aux grands procès, aux condamnations de masse spectaculaires d'hérétiques formels. Prime l'action en profondeur, l'humble recueil de l'information, l'avertissement discret, la réconciliation secrète, la mobilisation du clergé et des confesseurs pour surveiller et corriger ce qui doit l'être au coup par coup, au ras du sol. C'est à l'évidence des actions de ce type que pratiquent, et pratiquent de plus en plus, les inquisiteurs de M. Boeglin. L'inquisition espagnole n'a jamais été envisagée sous cet angle. Il y a là un magnifique terrain de recherche. Qui le premier, s'y lancera?

Ceci dit, M. Boeglin, qui s'est en outre distingué ces derniers temps par quelques bons articles et par une bonne édition de la Memoria histórica de Llorente ${ }^{4}$ compte désormais au rang des meilleurs spécialistes de l'inquisition espagnole.

Jean Pierre DEDIEU

Catherine Desos, La vie du R. P. Guillaume Daubenton S.J. (1648-1723). Un Jésuite fançais à la cour d'Espagne et à Rome. - Cordoue, Universidad de Córdoba, 2005, 225 p. - ISBN 84.7801.800.X.

Este libro ha sido sacado de una tesina. Pocas veces alcanzan tales trabajos el valor intrínseco necesario. En el presente caso no hay lugar a dudas: se trata de una aportación sobre el confesor que merece la atención de cualquier estudioso.

El confesor regio ha sido objeto, estos últimos ańos, de un renovado interés. Muy merecidamente. A la vez ministro de los cultos en una época en la que la regulación de las relaciones entre la sociedad eclesiástica y la civil es la primera función del Estado, y confidente íntimo del rey, acumula un poder que, por desenvolverse en gran parte a la sombra del secreto de confesión y

4. Juan Antonio Llorente ; Michel Boeglin, ed., España y la Inquisición. Memoria histórica... acerca del tribunal de la Inquisición seguida de Carta al señor Clausel de Courssergues sobre la Inquisición española, Sevilla, Renacimiento, 2007 [1811], 258 p. 